\title{
Research on Direct Model Reference-Based Adaptive Controller to Hand Tremors Reduction
}

\author{
Meysam Esmaeili, Farzin Piltan, Mohammad Ali Tayebi, Mahsa Piltan and \\ Mojtaba Yaghoot \\ Intelligent System and Robotic Lab, Iranian Institute of Advance Science and \\ Technology (IRAN SSP), Shiraz/Iran \\ piltan_f@iranssp.com,www.iranssp.com/english
}

\begin{abstract}
A tremor is an involuntary, somewhat rhythmic, muscle contraction and relaxation involving to and from movements (oscillations or switching) of one or more body parts. It is the most common of all involuntary movements and can affect the hands, arms, eyes, face, head, vocal folds, trunk, and legs. Most tremors occur in the hands. In some people, tremor is a symptom of another neurological disorder. This research focuses on the design and analysis of a model reference-based controller for highly nonlinear dynamic multi degrees of freedom, in presence of hand tremors. In order to provide a high performance nonlinear methodology, the inverse dynamical controller is selected. The inverse dynamic controller is the type of model reference controller. It is nonlinear and stable controller. To reduce the challenges of this controller hybrid fuzzy controller is used. However, this method has many advantages, but it has challenged in presence of uncertainties. To solve this challenge model reference-based direct adaptive methodology is used and applied to hybrid inverse dynamic fuzzy controller.
\end{abstract}

Keywords: hand tremors, inverse dynamic controller, fuzzy control, Multi degrees of freedom joints, Model reference-based adaptive control, hybrid control, direct adaptive control

\section{Introduction}

Tremor can be a symptom associated with disorders in those parts of the brain that control muscles throughout the body or in particular areas, such as the hands. Neurological disorders or conditions that can produce tremor, including multiple sclerosis, stroke, traumatic brain injury, chronic kidney disease and a number of neurodegenerative diseases that damage or destroy parts of the brainstem or the cerebellum, Parkinson's disease being the one most often associated with tremor. Other causes include the use of drugs (such as amphetamines, cocaine, caffeine, corticosteroids, SSRI), alcohol, mercury poisoning; or the withdrawal of drugs such as alcohol or benzodiazepine. Tremors can also be seen in infants with phenylketonuria (PKU), overactive thyroid or liver failure. Tremors can be an indication of hypoglycemia, along with palpitations, sweating and anxiety. Tremor can also be caused from lack of sleep, lack of vitamins, or increased stress. Deficiencies of magnesium and thiamine have also been known to cause tremor or shaking, which resolves when the deficiency is corrected. Some forms of tremor are inherited and run in families, while others have no known cause. Characteristics may include a rhythmic shaking in the hands, arms, head, legs, or trunk; shaky voice; difficulty writing or drawing; or problems holding and controlling utensils, such as a fork. Some tremors may be triggered by or become exaggerated during times of stress or strong emotion, when the individual is physically exhausted, or during certain postures or movements. Tremor may occur at any age, but is most common in middle-age and older persons, typically around the age of 52-61. It may be occasional, 
temporary, or occur intermittently. Tremor affects men and women equally. Regarding to above, age is the most important factor to hand tremors therefore, to reduce the effect of hand tremors in surgery, surgical robot was introduced. One of the main challenges in surgical robot was actuators. In this research multi degree of freedom actuator (MDFA) is used as a flexible actuator.

Multi degrees of freedom actuator (MDFA) are a type of nonlinear joints. These actuators are finding wide use in a number of Industries such as aerospace, Industrial medical and automotive. For high precession trajectory planning and control, it is necessary to replace the actuator system made up of several single-DOF motors connected in series and/or parallel with a single multi-DOF actuator [1-3]. The spherical motor (MDFA) has potential contributions to a wide range of applications such as coordinate measuring, object tracking, material handling, automated assembling, welding and laser cutting [8]. All these applications require high precision motion and fast dynamic response, which the spherical motor is capable of delivering [9-10]. The spherical motor exhibits coupled, nonlinear and very complex dynamics. The design and implementation of feedback controllers for the motor are complicated. The controller design is further complicated by the orientation-varying torque generated by the spherical motor [11]. One of the significant challenges in control algorithms is a linear behavior controller design for nonlinear systems. Some of MDFA which work in industrial processes are controlled by linear PID controllers, but the design of linear controllers for MDFA is extremely difficult in presence of hand tremors. To solve this challenge, the nonlinear model reference controller is a candidate.

One of the powerful types of nonlinear controller is an inverse dynamic controller. This controller is used to control of highly nonlinear systems. It is based on feedback linearization and computes the required torques using the nonlinear feedback control law. This controller works very well when all dynamic and physical parameters are known, but when the system has variation in dynamic parameters, the controller has no acceptable performance. In practice, physical system parameters are unknown or time variant, therefore, hybrid inverse dynamic like controller used to compensate dynamic equation.

To improve the robustness in inverse dynamic controller, hybrid fuzzy logic controller is introduced. Fuzzy logic theory is used to remove the system's dynamics. Although the fuzzy-logic control is not a new technique, its application in this current research is considered to be novel since it aimed for an automated dynamic-less response rather than for the traditional objective of uncertainties compensation [12-14]. The intelligent tracking control using the fuzzy-logic technique provides a cost-and-time efficient control implementation due to the automated dynamic-less input. This in turn would further inspire multi-uncertainties testing for continuum robot [11]. In the project we can use fuzzy logic theory when a plant can be considered as a black box with outputs available for measurement and a possibility of changing inputs. The plant is supposed to be observable and controllable. Some information about the plant operation or plant control is available, which can or cannot be a quantitative nature, but it can be formulated as a set of rules (maybe after some processing). An acceptable fuzzy control solution is possible, which should satisfy design specifications. It must not be optimal with regard to any criteria as it is hard to prove that a fuzzy control system is optimized and even stable. However, a fuzzy controller is able to provide a stable and 'good' solution. However, hybrid inverse dynamic like controller can improve the stability and limitation robustness, but it has limitation in uncertain condition (hand tremors). To improve this challenge model reference-based direct adaptive methodology is introduced.

In various dynamic parameter systems that need to be training on-line adaptive control methodology is used. The adaptive control methodology can be classified into two main groups, namely, traditional adaptive method and fuzzy adaptive method. Model reference variable structure adaptive method is used in systems which want to tune parameters by system's dynamic knowledge. This paper is organized as follows; section 2, has served as 
an introduction to the dynamic of three degrees of freedom spherical motor. Part 3, introduces and describes the methodology algorithm. Section 4 presents the simulation results and discussion of this algorithm applied to three degrees of freedom spherical motor and the final section describe the conclusion.

\section{Theory}

Dynamic modeling of spherical motors is used to describe the behavior of the spherical motor such as linear or nonlinear dynamic behavior, design of the model based controller such as a pure sliding mode controller which design this controller is based on nonlinear dynamic equations, and for simulation. The dynamic model describes the relationship between motion, velocity, and accelerations to force/torque or current/voltage and also it can be used to describe the particular dynamic effects (e.g., inertia, coriolios, centrifugal, and the other parameters) to behavior of the system. Spherical motor is nonlinear and uncertain dynamic parameters and it is 3 degrees of freedom (DOF) electrical motor.

The equation of a spherical motor governed by the following equation:

$$
H(q)\left[\begin{array}{c}
\ddot{\alpha} \\
\ddot{\beta} \\
\ddot{\gamma}
\end{array}\right]+\mathbf{B}(\boldsymbol{q})\left[\begin{array}{c}
\dot{\alpha} \dot{\beta} \\
\dot{\alpha} \dot{\gamma} \\
\dot{\boldsymbol{\beta}} \dot{\gamma}
\end{array}\right]+\boldsymbol{C}(\boldsymbol{q})\left[\begin{array}{c}
\dot{\alpha}^{2} \\
\dot{\boldsymbol{\beta}}^{2} \\
\dot{\gamma}^{2}
\end{array}\right]=\left[\begin{array}{c}
\tau_{x} \\
\tau_{y} \\
\tau_{z}
\end{array}\right]
$$

Where $\tau$ is actuation torque, $\mathrm{H}(\mathrm{q})$ is a symmetric and positive definite inertia matrix, B (q) is the matrix of coriolios torques, $\mathrm{C}(\mathrm{q})$ is the matrix of centrifugal torques.

This is a decoupled system with simple second order linear differential dynamics. In other words, the component $\ddot{q}$ Influences, with a double Integrator relationship, only the variable $q_{i}$, independently of the motion of the other parts. Therefore, the angular acceleration is found as to be:

$$
\ddot{q}=H^{-1}(q) .\{\tau-\{B+C\}\}
$$

This technique is very attractive from a control point of view.

Study of spherical motor is classified into two main groups: kinematics and dynamics. Calculate the relationship between rigid bodies and final part without any forces is called Kinematics. Study of this part is pivotal to design with an acceptable performance controller, and in real situations and practical applications. As expected the study of kinematics is divided into two main parts: forward and inverse kinematics. Forward kinematics have been used to find the position and orientation of task frame when angles of joints are known. Inverse kinematics has been used to find possible joints variable (angles) when all position and orientation of task frame be active [1].

According to the forward kinematics formulation;

$$
\boldsymbol{\Psi}(\boldsymbol{X}, \boldsymbol{q})=\mathbf{0}
$$

Where $\Psi(.) \in R^{n}$ is a nonlinear vector function, $X=\left[X_{1}, X_{2}, \ldots \ldots, X_{l}\right]^{T}$ is the vector of task space variables which generally task frame has three task space variables, three orientation, $q=\left[q_{1}, q_{2}, \ldots, q_{n}\right]^{T}$ is a vector of angles or displacement, and finally $n$ is the number of actuated joints. The Denavit-Hartenberg $(\mathrm{D}-\mathrm{H})$ convention is a method of drawing spherical motor free body diagrams. Denvit-Hartenberg (D-H) convention study is necessary to calculate forward kinematics in this motor.

A systematic Forward Kinematics solution is the main target of this part. The first step to compute Forward Kinematics (F.K) is finding the standard D-H parameters. The following steps show the systematic derivation of the standard D-H parameters.

1. Locate the spherical motor

2. Label joints

3. Determine joint rotation $(\theta)$ 
4. Set up base coordinate frames.

5. Setup joints coordinate frames.

6. Determine $\alpha_{i}$, that $\alpha_{i}$, link twist, is the angle between $Z_{i}$ and $Z_{i+1}$.

7. Determine $d_{i}$ and $a_{i}$, that $a_{i}$, link length, is the distance between $Z_{i}$ and $Z_{i+1}$ along $X_{i}$. $d_{i}$, offset, is the distance between $X_{i-1}$ and $X_{i}$ along $Z_{i}$ axis.

8. Fill up the D-H parameters table. The second step to compute Forward kinematics is finding the rotation matrix $\left(R_{n}^{0}\right)$. The rotation matrix from $\left\{F_{i}\right\}$ to $\left\{F_{i-1}\right\}$ is given by the following equation;

$\boldsymbol{R}_{i}^{i-1}=\boldsymbol{U}_{\boldsymbol{i}\left(\theta_{i}\right)} \boldsymbol{V}_{\boldsymbol{i}\left(\boldsymbol{\alpha}_{\boldsymbol{i}}\right)}$

Where $U_{i\left(\theta_{i}\right)}$ is given by the following equation;

$$
U_{i\left(\theta_{i}\right)}=\left[\begin{array}{ccc}
\cos \left(\theta_{i}\right) & -\sin \left(\theta_{i}\right) & 0 \\
\sin \left(\theta_{i}\right) & \cos \left(\theta_{i}\right) & 0 \\
0 & 0 & 1
\end{array}\right]
$$

and $V_{i\left(\alpha_{i}\right)}$ is given by the following equation;

$$
V_{i\left(\theta_{i}\right)}=\left[\begin{array}{ccc}
1 & 0 & 0 \\
0 & \cos \left(\alpha_{i}\right) & -\sin \left(\alpha_{i}\right) \\
0 & \sin \left(\alpha_{i}\right) & \cos \left(\alpha_{i}\right)
\end{array}\right]
$$

So $\left(R_{n}^{0}\right)$ is given by [8]

$$
R_{n}^{\mathbf{0}}=\left(U_{1} V_{1}\right)\left(U_{2} V_{2}\right) \ldots \ldots\left(U_{n} V_{n}\right)
$$

The final step to compute the forward kinematics calculates the transformation ${ }_{n}^{0} T$ by the following formulation [3]

$$
{ }_{n}^{0} T={ }_{1}^{0} T \cdot{ }_{2}^{1} T \cdot{ }_{3}^{2} T \ldots \ldots . .{ }_{n}^{n-1} T=\left[\begin{array}{cc}
R_{n}^{0} & 0 \\
0 & 1
\end{array}\right]
$$

\section{Methodology}

Inverse dynamics control is based on cancelling decoupling and nonlinear terms of the dynamics of joints. The inverse dynamics control has the form:

$$
\boldsymbol{\tau}=\boldsymbol{H}(\boldsymbol{q}) \cdot \boldsymbol{V}+\boldsymbol{B}(\boldsymbol{q})[\dot{\boldsymbol{q}} \dot{\boldsymbol{q}}]+\boldsymbol{C}(\boldsymbol{q})[\dot{\boldsymbol{q}}]^{2}
$$

where typical choices for $V$ are:

$$
\boldsymbol{V}=\ddot{\boldsymbol{q}}_{\boldsymbol{d}}+\boldsymbol{K}_{v}\left(\dot{\boldsymbol{q}}_{d}-\dot{\boldsymbol{q}}_{a}\right)+\boldsymbol{K}_{\boldsymbol{p}}\left(\boldsymbol{q}_{d}-\boldsymbol{q}_{a}\right)
$$

or with an integral term

$$
v=\ddot{q}_{d}+K_{v}\left(\dot{q}_{d}-\dot{q}_{a}\right)+K_{p}\left(q_{d}-q_{a}\right)+K_{I} \int\left(q_{d}-q_{a}\right) d t
$$

where $e=\left(q_{d}-q_{a}\right)$, the resulting error dynamics is

$$
\dddot{q}_{d}+K_{v} \ddot{e}+K_{p} \dot{e}+K_{I} e=0
$$

where $\boldsymbol{K}_{\boldsymbol{p}}, \boldsymbol{K}_{\boldsymbol{v}}$ and $\boldsymbol{K}_{\boldsymbol{I}}$ are the controller gains. Based on (12) in inverse dynamic controller; the performance depends on the gain updating factor $(\boldsymbol{K})$ : derivative gain updating factor $\left(\boldsymbol{K}_{\boldsymbol{v}}\right)$, proportional gain updating factor $\left(\boldsymbol{K}_{\boldsymbol{p}}\right)$ and integral gain updating factor $\left(\boldsymbol{K}_{\boldsymbol{I}}\right)$.

Regarding to (9) this controller has a challenge in the presence of uncertainty (hand tremors). To reduce this challenge fuzzy logic controller is used as an estimator. In this research hybrid fuzzy methodology is used beside the inverse dynamic methodology. In this part we describe the basic definitions of fuzzy sets and operators on fuzzy sets. Regarding to Figure 1, design fuzzy logic controller is divided into three stages:

- Fuzzification

- Fuzzy Processing

- Defuzzification Method 


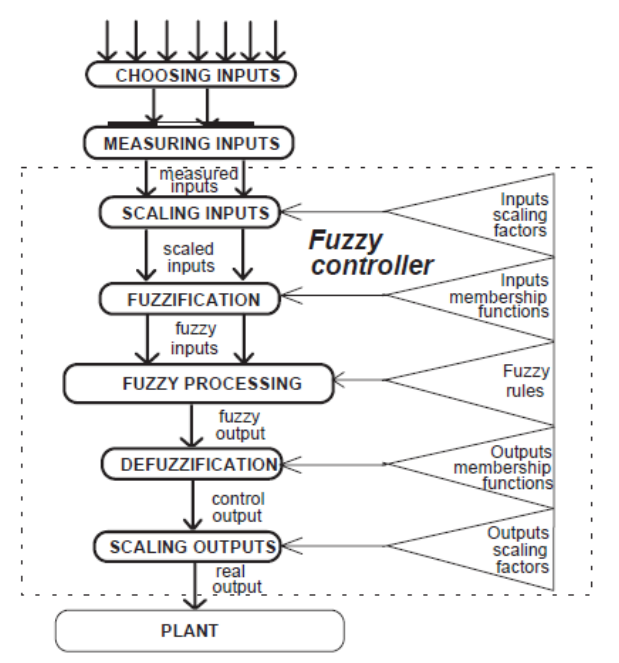

Figure 1. Fuzzy Controller Operation

The first step focuses on the fuzzification. This part has three subparts: choosing inputs, measuring inputs and scaling inputs. In this research PD fuzzy logic controller is used. In our research, we expect inputs to take values from the constrained voltage range that defines input domains $\mathrm{e} \in[-200,200]$ and $\Delta \mathrm{e} \in[-30,30]$ from the characteristics of the system design. Over these domains, a fuzzy controller has seven linearly distributed triangular fuzzy sets for both inputs, as shown in Figure 2. The input values that exceed the limits of the specified input domains belong only to boundary fuzzy sets with the maximum degree of membership.

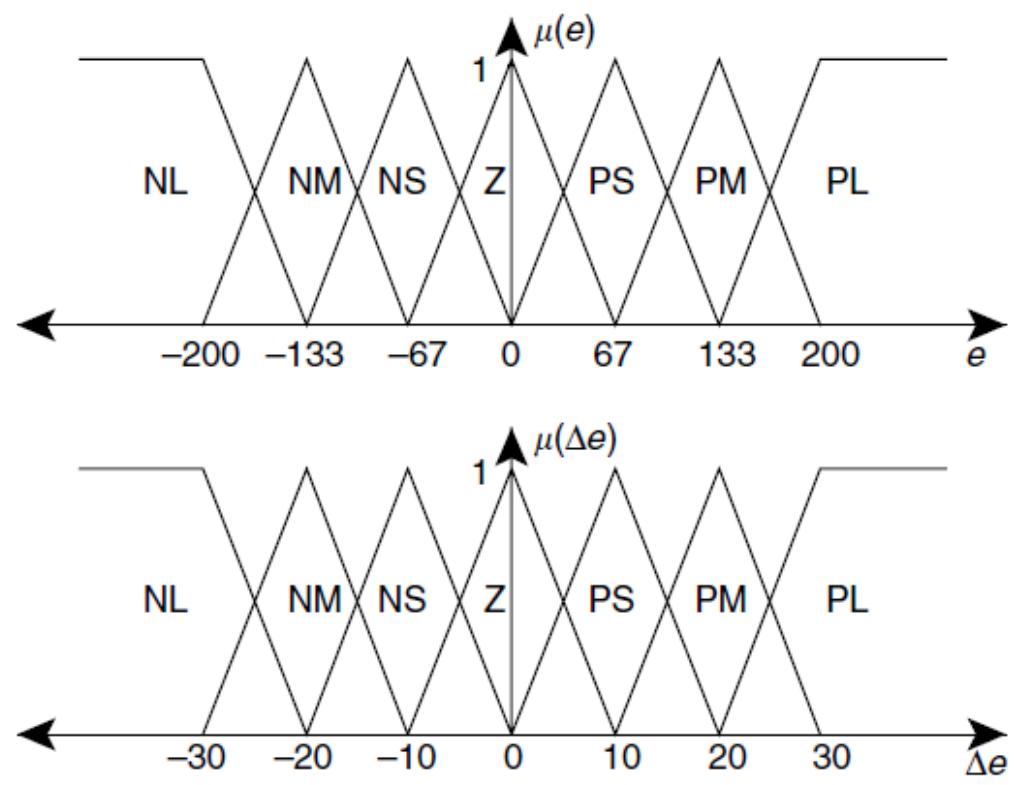

Figure 2. The Distribution of Fuzzy Membership Function

The fuzzy inference engine offers a mechanism for transferring the rule base in fuzzy set which it is divided into two most important methods, namely, Mamdani method and Sugeno method. Mamdani method is one of the common fuzzy inference systems and he designed one of the first fuzzy controllers to control of system engine. Mamdani's fuzzy inference system is divided into four major steps: fuzzification, rule evaluation, aggregation of the rule outputs and defuzzification. Michio Sugeno uses a singleton as a 
membership function of the rule consequent part. The following definition shows the Mamdani and Sugeno fuzzy rule base

\section{if $x$ is $A$ and $y$ is $B$ then $z$ is $C$ 'mamdani' \\ if $x$ is $A$ and $y$ is $B$ then $z$ is $f(x, y)$ 'sugeno'}

When $x$ and $y$ have crisp values fuzzification calculates the membership degrees for antecedent part. Rule evaluation focuses on fuzzy operation $(A N D / O R)$ in the antecedent of the fuzzy rules. The aggregation is used to calculate the output fuzzy set and several methodologies can be used in fuzzy logic controller aggregation, namely, Max-Min aggregation, Sum-Min aggregation, Max-bounded product, Max-drastic product, Maxbounded sum, Max-algebraic sum and Min-max. Two most common methods that used in fuzzy logic controllers are Max-min aggregation and Sum-min aggregation. Max-min aggregation defined as below;

$$
\mu_{U}\left(x_{k}, y_{k}, U\right)=\mu_{\cup i=1}^{r} F R^{i}\left(x_{k}, y_{k}, U\right)=\max \left\{\min _{i=1}^{r}\left[\mu_{R p q}\left(x_{k}, y_{k}\right), \mu_{p_{m}}(U)\right]\right\}
$$

The Sum-min aggregation defined as below

$$
\mu_{U}\left(x_{k}, y_{k}, U\right)=\mu_{\cup i=1}^{r} F R^{i}\left(x_{k}, y_{k}, U\right)=\sum \min _{i=1}^{r}\left[\mu_{R p q}\left(x_{k}, y_{k}\right), \mu_{p_{m}}(U)\right]
$$

where $r$ is the number of fuzzy rules activated by $x_{k}$ and $y_{k}$ and also $\mu_{\cup_{i=1}^{r} F R^{i}}\left(x_{k}, y_{k}, U\right)$ is a fuzzy interpretation of $i-t h$ rule. Defuzzification is the last step in the fuzzy inference system which it is used to transform fuzzy set to crisp set. Consequently defuzzification's input is the aggregate output and the defuzzification's output is a crisp number. Center of gravity method (COG) and Centre of area method $(C O A)$ are two most common defuzzification methods, which $C O G$ method used the following equation to calculate the defuzzification

$$
\operatorname{COG}\left(x_{k}, y_{k}\right)=\frac{\sum_{i} U_{i} \sum_{j=1}^{r} \cdot \mu_{u}\left(x_{k}, y_{k}, U_{i}\right)}{\sum_{i} \sum_{j=1}^{r} \cdot \mu_{u}\left(x_{k}, y_{k}, U_{i}\right)}
$$

and $C O A$ method used the following equation to calculate the defuzzification

$$
\operatorname{COA}\left(x_{k}, y_{k}\right)=\frac{\sum_{i} U_{i} \cdot \mu_{u}\left(x_{k}, y_{k}, U_{i}\right)}{\sum_{i} \mu_{U} \cdot\left(x_{k}, y_{k}, U_{i}\right)}
$$

Where $\operatorname{COG}\left(x_{k}, y_{k}\right)$ and $\operatorname{COA}\left(x_{k}, y_{k}\right)$ illustrates the crisp value of defuzzification output, $U_{i} \in U$ is da discrete elementof an output of the fuzzy set, $\mu_{U}$. $\left(x_{k}, y_{k}, U_{i}\right)$ is the fuzzy set membership function, and $r$ is the number of fuzzy rules. Figure 3 shows the hybrid inverse dynamic like controller.

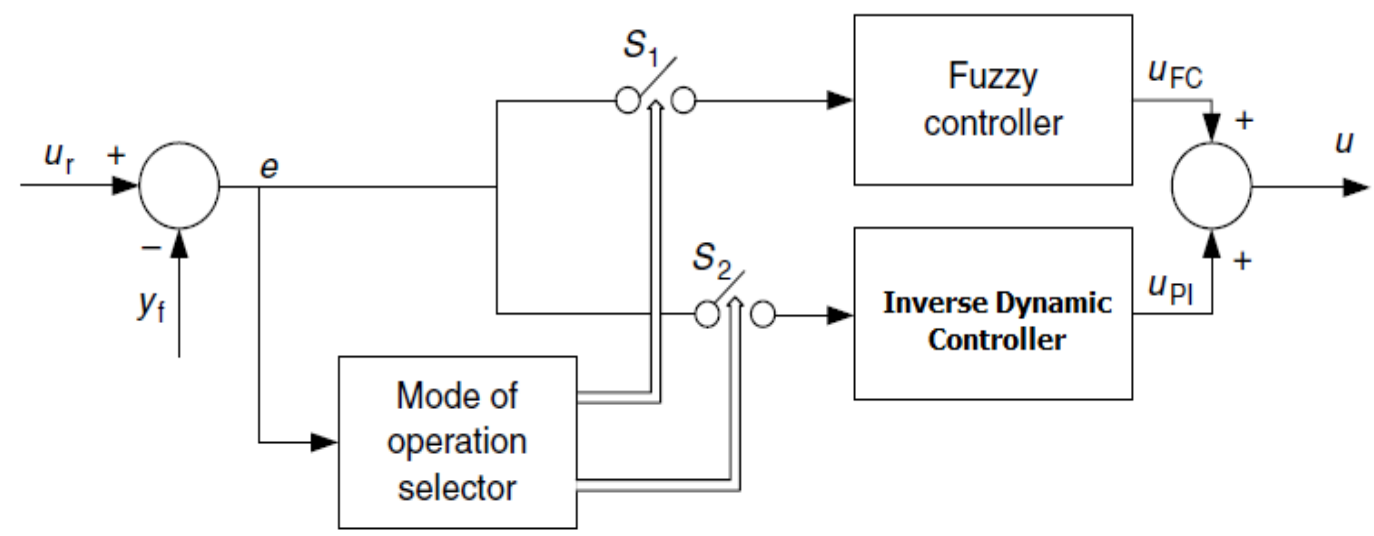

Figure 3. Hybrid Inverse Dynamic like Controller

Regarding to this design it has two important challenges: chattering based on mode operation selector and robustness. To improve these two challenges direct model reference adaptive controller is designed in this research. An adaptive control usually 
supposes modifications of the plant model, and a learning control assumes the modification of the control strategy based on past experience. One should note that these definitions are general and not specific to a fuzzy controller. Any adaptive control system can adjust itself to accommodate new situations, such as changes in the observed dynamic behavior of a plant. In essence, adaptive techniques monitor the input-output behavior of the plant to identify, either explicitly or implicitly, the parameters of an assumed dynamic model. The control system parameters are then adjusted to achieve some desired performance objectives. Thus, adaptive techniques seek to achieve an increased performance by updating or refining some representation, which is determined (in whole or in part) by a model of the plant structure, based on on-line measurement information. An adaptive control system will attempt to adapt, if the behavior of the plant changes by a significant degree.

The problem is time for adaptation. If the dynamic characteristics of the plant vary considerably over its operation (e.g., due to nonlinear dynamics), then the control system may be required to adapt continually. During these adaptation periods, a high closed-loop performance cannot be guaranteed. Note that this adaptation can occur even in the absence of time-varying dynamics and disturbances, since the control system must readapt every time a different dynamic regime is encountered (i.e., one for which the current control law is inadequate), even if it is returning to an operating condition it has encountered and successfully adapted to before. So the adaptation must take place in every case of changing. This inefficiency results in degraded performance, since the transient behavior due to the parameter adjustment will occur every time the recently observed dynamic behavior of the plant changes by a sufficient degree. In general, adaptive controllers operate by optimizing a small set of adjustable parameters to account for a plant behavior that is local in both state-space and time. To be effective, adaptive controllers must have relatively fast dynamics so that they can react quickly to any change of the plant behavior. In some instances, the plant parameters may vary so fast (perhaps due to nonlinearity), that the adaptive system cannot maintain desired performance through an adaptive action alone. Because the learning system retains some information, or we may say, some knowledge, it can in principle react more rapidly to spatial variations once it has learned. Figure 4 shows the general structure of adaptive control systems.

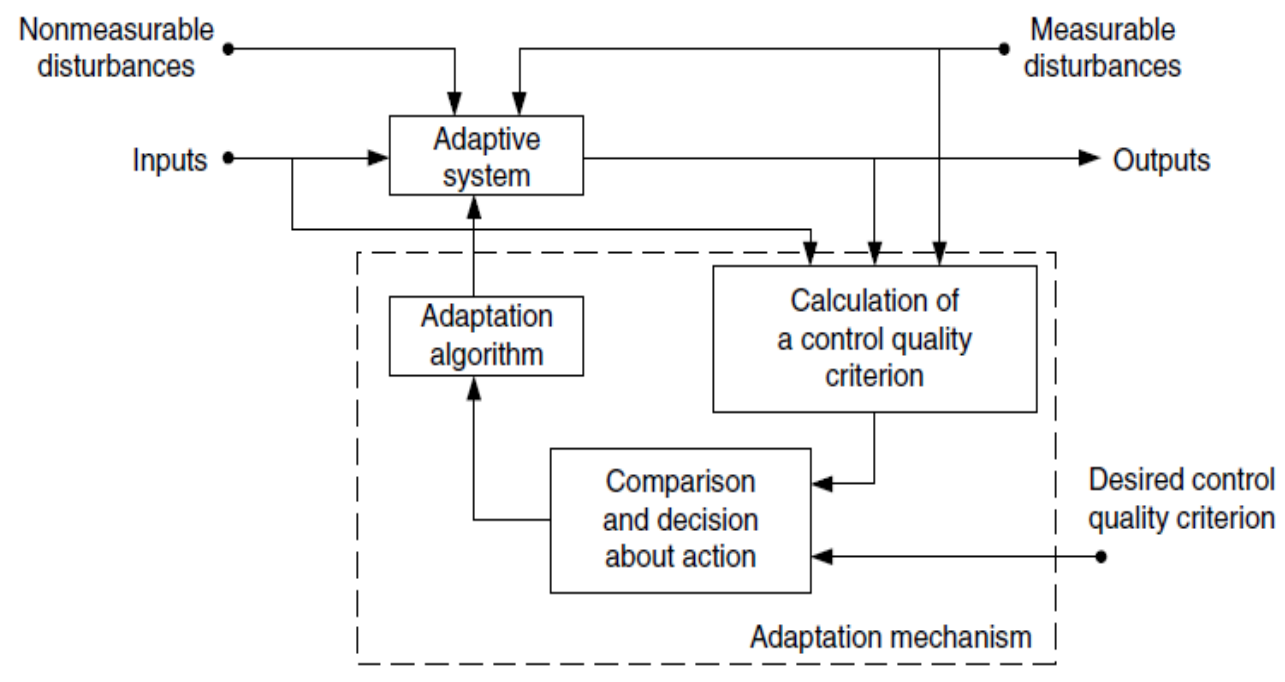

Figure 4. General Structure of Adaptive Control Systems 
In this research, direct adaptive model-base is applied to inverse dynamic like controller. Figure 5 shows the model reference-based adaptive controller with parameter adaptation.

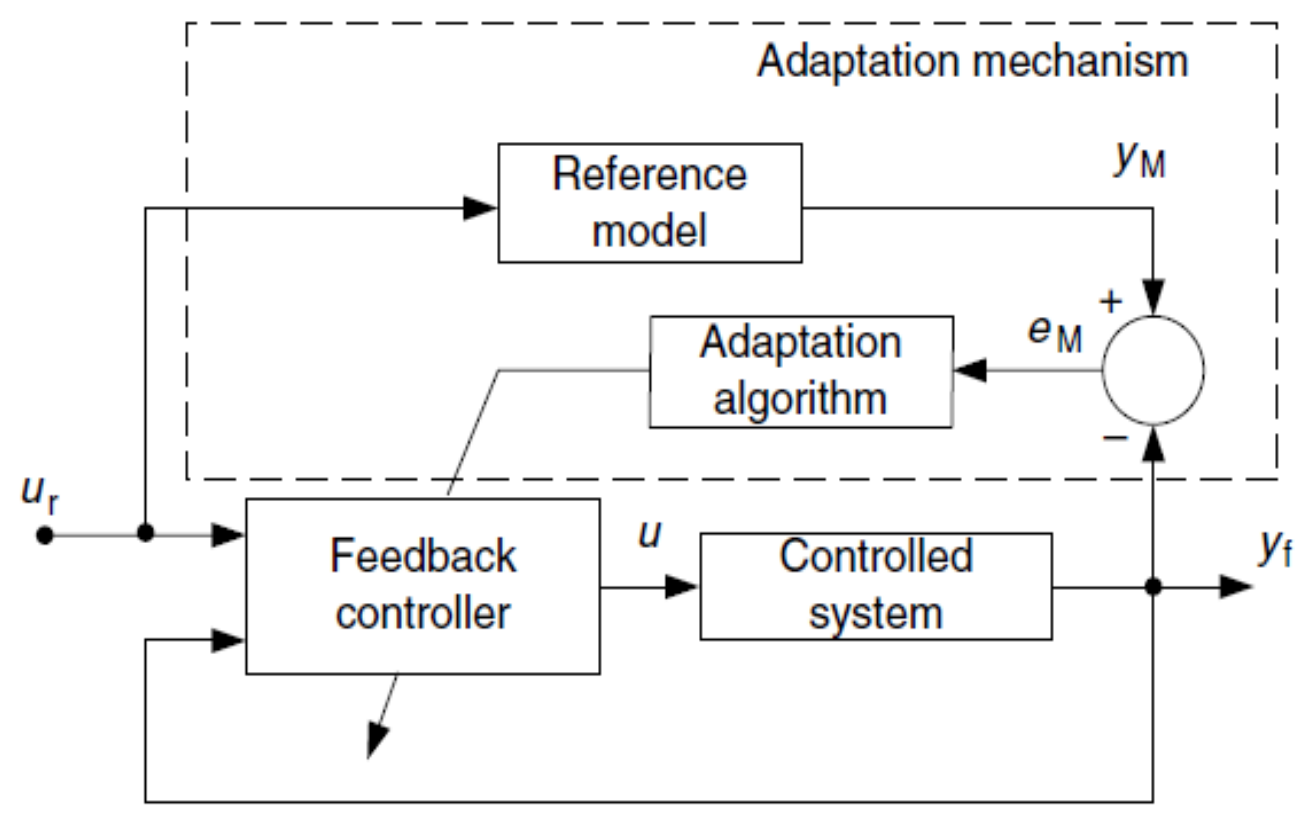

Figure 5. Model Reference-Based Adaptive Control System with Parameter Adaption

Regarding to Figure 5, dynamic modeling of inverse dynamic controller is used as adaptive part. The part of adapting algorithm in this research is designed based on fuzzy logic algorithm. According to proposed method, modify robust inverse dynamic controller is designed and applied to MDFA system. This method is robust and stable in presence of hand tremors.

\section{Results and Discussion}

Inverse dynamic controller and proposed methodology are compared. This simulation is used to control position of three degrees of freedom spherical motor without and with external disturbance. The simulation was implemented in MATLAB/SIMULINK environment. These systems are tested by band limited white noise with a predefined $40 \%$ of relative to the input signal amplitude. This type of noise is used to external disturbance in continuous and hybrid systems and applied to nonlinear dynamic of these controllers.

Certain Tracking Data: Figure 6 shows the tracking data in certain parameters. Regarding the following Figure, the inverse dynamic controller has about 3\% overshoot. This item caused by moderate vibration. Proposed method removes overshoot, however the rate of trajectory following decrease compared to inverse dynamic control. According to following graph, both methods have the same steady state error near to the zero. 


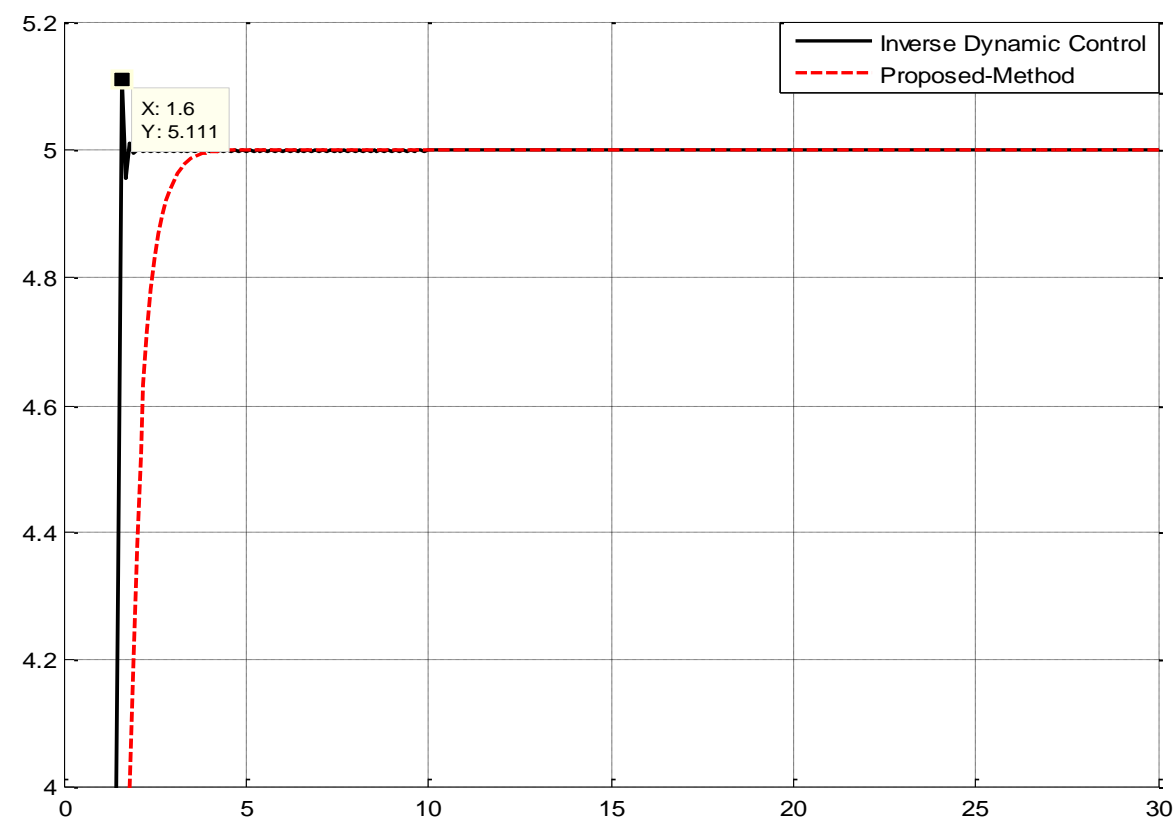

Figure 6. Data Tracking Performance: Certain Condition

Certain Torque Performance: Torque performance has an essential role to regulate the energy consumption. This item also is very important to motor vibration. Regarding to Figure 7 both techniques have the same torque performance in certain condition. According to Figures 6and 7 however, the proposed method can eliminate the overshoot, but inverse dynamic technique also has accepted performance.

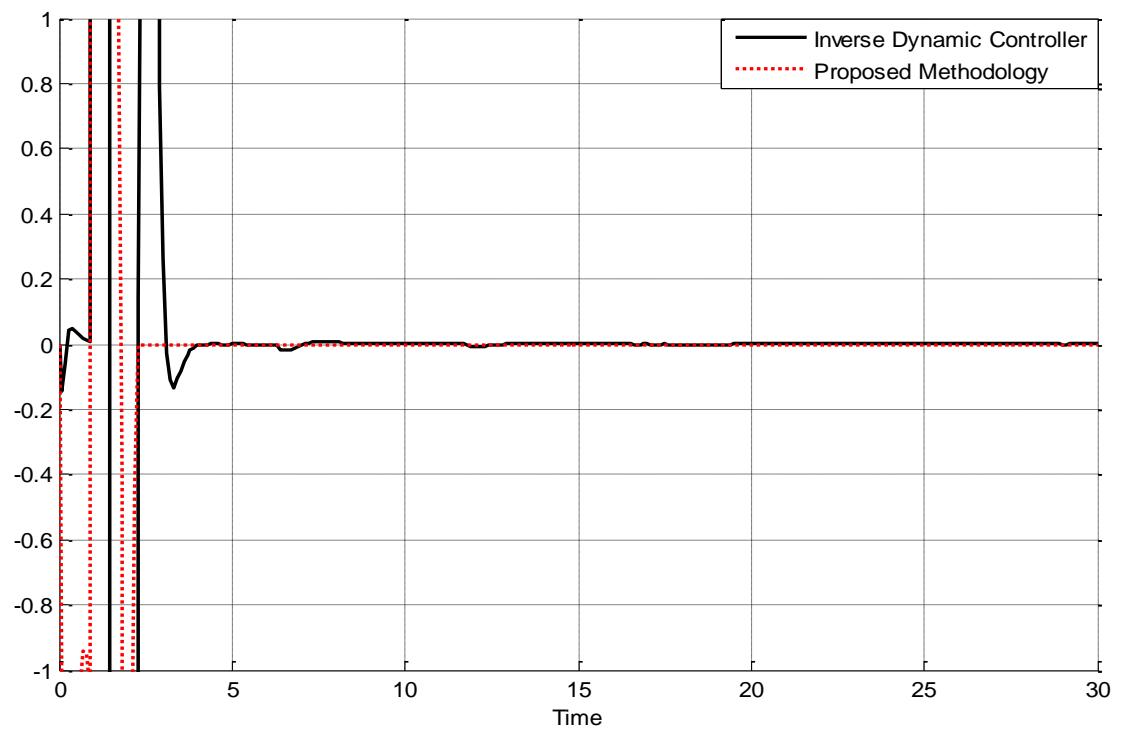

Figure 7. Torque Performance: Certain Condition

Power of Disturbance Rejection: To robustness checking, both techniques are tested in uncertain conditions. In this research uncertain condition meant by hand tremors. Regarding to Figure 8, the inverse dynamic controller is not robust against to hand tremors. This controller has large fluctuations which caused to instability. Conversely, the 
proposed method is very robust. However, in certain condition both control methods have the same performance, but in uncertainty inverse dynamic controller has many challenges.

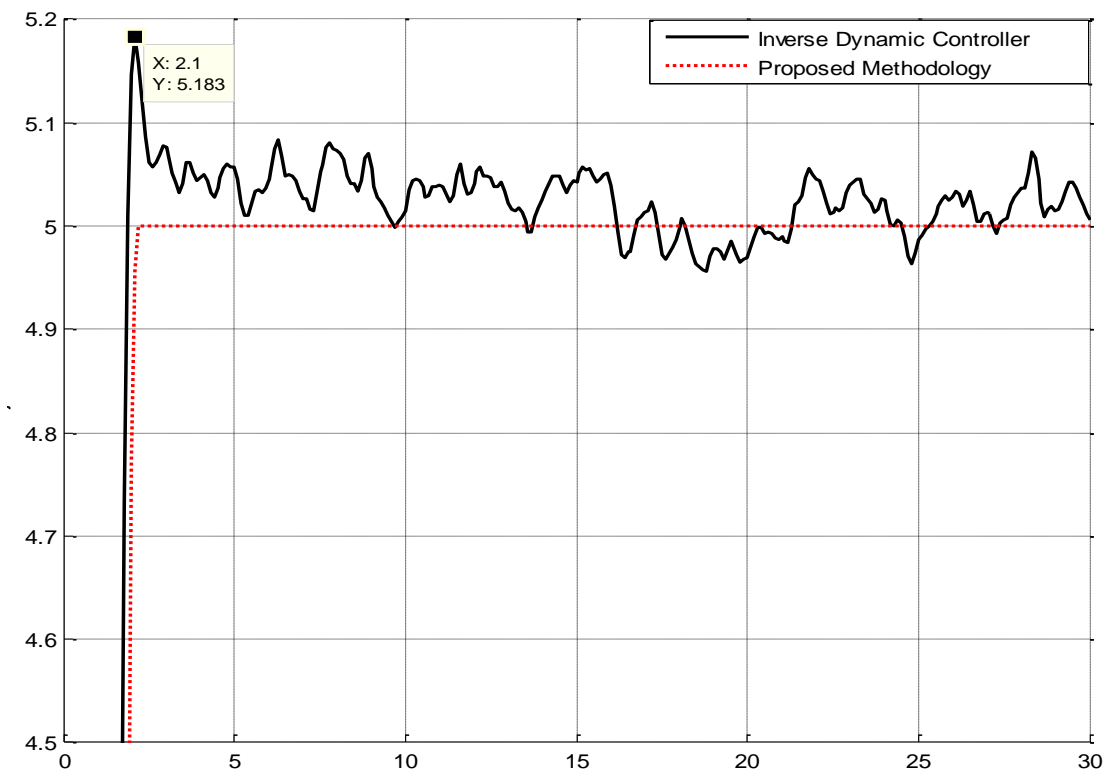

Figure 8. Data Tracking Performance: Uncertain Condition

Uncertain Torque Performance: Figure 9 shows the uncertain (hand tremors) torque performance. According to below graph, the proposed method has stayed stable torque performance. This item caused to eliminate the hand tremors as well as reduce energy consumption. By contrast, torque performance in pure inverse dynamic controller fell erratically from 0.2 to -0.2 and ceased to have a vibration in multi degrees of freedom joints as well increase the energy consumption.

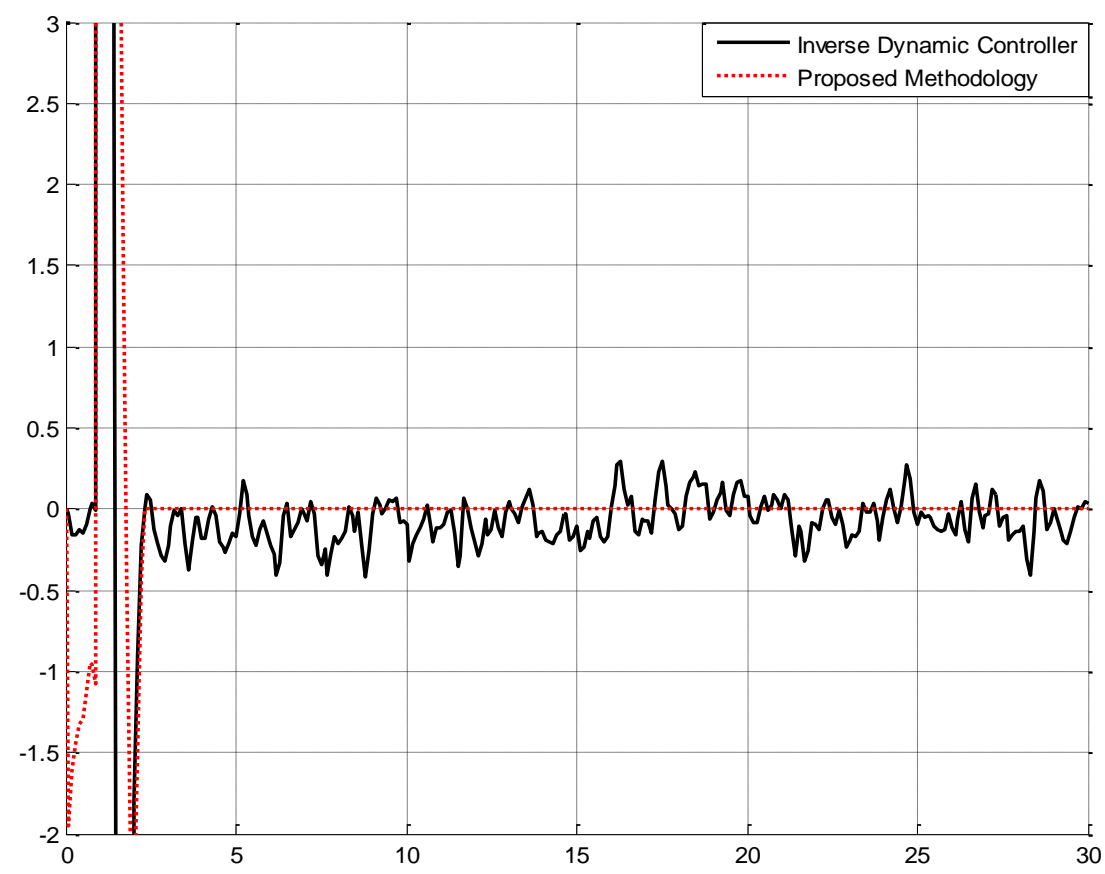

Figure 9. Torque Performance: Uncertain Condition 


\section{Conclusion}

In this research the challenge of hand tremors eliminated based on intelligent inverse dynamic control technique. Multi degrees of freedom actuators used in many applications such as medical industry, but they are too sensitive. These devices are nonlinear, MIMO and coupling effect, therefore design a robust controller can be an important challenge. The inverse dynamic controller is a nonlinear controller, to improve the challenge of model-reference controller, fuzzy logic controller is used as an estimator. Both inverse dynamic controller and fuzzy based inverse dynamic controller have the same challenge, namely; robustness. To solve the robustness challenge, direct model base adaptive inverse dynamic like controller is introduced in this research. Regarding to the result of this research, the proposed method can solve the challenges of stability and robustness.

\section{Acknowledgement}

The authors would like to thank the anonymous reviewers for their careful reading of this paper and for their helpful comments. This work was supported by the Iranian Institute of Advance Science and Technology Program of Iran under grant no. 2013-Persian Gulf-2A.

Iranian center of Advance Science and Technology (IRAN SSP) is one of the independent research centers specializing in research and training across of Control and Automation, Electrical and Electronic Engineering, and Mechatronics \& Robotics in Iran. At IRAN SSP research center, we are united and energized by one mission to discover and develop innovative engineering methodology that solve the most important challenges in field of advance science and technology. The IRAN SSP Center is instead to fill a long standing void in applied engineering by linking the training a development function one side and policy research on the other. This center divided into two main units:

- $\quad$ Education unit

- $\quad$ Research and Development unit

\section{References}

[1] G. I. Vachtsevanos, K. Davey and K. M. Lee, "Development of a Novel Intelligent Robotic Manipulator," IEEE Control System Magazine, (1987), pp. 9-15.

[2] K. Davey, G. I. Vachtsevanos and R. Powers, "An analysis of Fields and Torques in Spherical Induction Motors," IEEE Transactions on Magnetics, vol. MAG-23, (1987), pp. 273-282.

[3] A. Foggia, E. Oliver and F. Chappuis, "New Three Degrees of Freedom Electromagnetic Actuator," Conference Record -lAS Annual Meeting, vol. 35, (1988), New York.

[4] K. M. Lee, G. Vachtsevanos and C.-K. Kwan, "Development of a Spherical Wrist Stepper Motor," Proceedings of the 1988 IEEE Intemational Conference on Robotics and Automation, Philadelphia, PA. April 26-29.

[5] K. M. Lee and I. Pei, "Kinematic Analysis of a Three Degree-of-Freedom Spherical Wrist Actuator," The Fifth International Conference on Advanced Robotics, Italy, (1991).

[6] I. Wang, G. Jewel and D. Howe, "Modeling of a Novel Spherical Pennanent Magnet Actuator," Proceedings of IEEE International Conference on Robotics and Automation, Albuquerque, New Mexico, (1997,) pp. 1190-1195.

[7] I. Wang, G. Jewel and D. Howe, "Analysis, Design and Control of a Novel Spherical Pennanent Magnet Actuator," IEE Proceedings on Electrical Power Applications., vol. 154, no. 1, (1998).

[8] G. S. Chirikjian and D. Stein, "Kinematic Design and Commutation of a Spherical Stepper Motor," IEEEIASME Transactions on Mechatronics, vol. 4, n 4, Piscataway, New Jersey, (1999) December, pp. 342-353.

[9] K. Kahlen and R. W. De Doncker, "CW'l'ent Regulators for Multi-phase Pennanent Magnet Spherical Machines", Industry Applications Conference Record of the 2000 IEEE, vol. 3, (2000), pp. 2011-2016.

[10] K. M. Lee, I. Pei and U. Gilboa, "On the Development of a Spherical Wrist Actuator," Proceedings of the 16th NSF Conference on Manufacturing Systems Research, Tempe AZ, (1990) January 8-12.

[11] C. Yang and Y. S. Back, "Design and Control of the 3-dcgnes of freedom actuator by Controlling the Electromagnetic Force," IEEE Transactions on Magnetics, (1999) May, pp. 3607-3609.

[12] A. Barzegar, F. Piltan, M. Vosoogh, A. M. Mirshekaran and A. Siahbazi, "Design Serial Intelligent Modified Feedback Linearization like Controller with Application to Spherical Motor", IJITCS, vol. 6, no. 5, (2014), pp. 72-83, DOI: 10.5815/ijitcs.2014.05.10. 
[13] M. Shamsodini, F. Piltan, S. Rahbar, E. Pooladi and H. Davarpanah, "Design New PID like Fuzzy CTC Controller: Applied to Spherical Motor", IJMECS, vol. 6, no. 5, (2014), pp. 60-68, DOI:10.5815/ijmecs.2014.05.08.

[14] M. Rahmani, F. Piltan, F. Matin, H. Cheraghi and N. Sobhani, "Design Intelligent System Compensator to Computed Torque Control of Spherical Motor", IJISA, vol. 6, no. 8, (2014), pp. 87-96, DOI:10.5815/ijisa.2014.08.10.

\section{Authors}

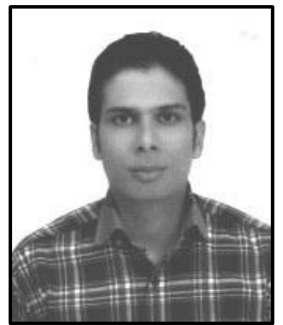

Meysam Esmaeili is currently a co researcher in the Intelligent System and Robotic Lab at Iranian Institute of Advance Science and Technology (IRAN SSP) of the program for design high precision and fast dynamic controller for multi-DOF- actuators.

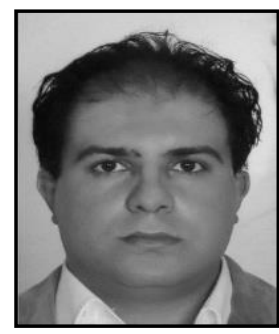

Farzin Piltan was born on 1975, Shiraz, Iran. In 2004 he is jointed Institute of Advance Science and Technology, Research and Development Center, IRAN SSP. Now he is a dean of Intelligent Control and Robotics Lab. He is led of team (47 researchers) to design and build of nonlinear control of industrial robot manipulator for experimental research and education and published about 54 Papers in this field since 2010 to 2012, team supervisor and leader ( 9 researchers) to design and implement intelligent tuning the rate of fuel ratio in internal combustion engine for experimental research and education and published about 17 Journal papers since 2011 to 2013, team leader and advisor (34 researchers) of filtering the hand tremors in flexible surgical robot for experimental research and education and published about 31 journal papers in this field since 2012 to date, led of team (21 researchers) to design high precision and fast dynamic controller for multi-degrees of freedom actuator for experimental research and education and published about 7 journal papers in this field since 2013 to date, led of team ( 22 researchers) to research of full digital control for nonlinear systems (e.g., Industrial Robot Manipulator, IC Engine, Continuum Robot, and Spherical Motor) for experimental research and education and published about 4 journal papers in this field since 2010 to date and finally led of team (more than 130 researchers) to implementation of Project Based-Learning project at IRAN SSP research center for experimental research and education, and published more than 110 journal papers since 2010 to date. In addition to 7 textbooks, Farzin Piltan is the main author of more than 115 scientific papers in refereed journals. $\mathrm{He}$ is editorial review board member for 'international journal of control and automation (IJCA), Australia, ISSN: 2005-4297; 'International Journal of Intelligent System and Applications (IJISA)', Hong Kong, ISSN:2074-9058; 'IAES international journal of robotics and automation, Malaysia, ISSN:2089-4856; 'International Journal of Reconfigurable and Embedded Systems', Malaysia, ISSN:2089-4864. His current 


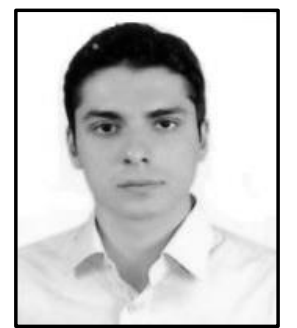

research interests are nonlinear control, artificial control system and applied to FPGA, robotics and artificial nonlinear control and IC engine modeling and control.

Mohammad Ali Tayebi is currently a co researcher in the Intelligent System and Robotic Lab at Iranian Institute of Advance Science and Technology (IRAN SSP) of the program for design high precision and fast dynamic controller for multi-DOFactuators.

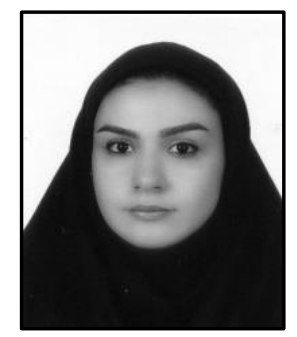

Mahsa Piltan is currently a co researcher in the Intelligent System and Robotic Lab at Iranian Institute of Advance Science and Technology (IRAN SSP) of the program for design high precision and fast dynamic controller for multi-DOF- actuators.

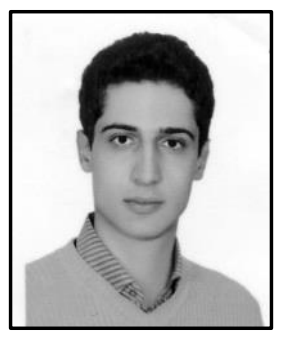

Mojtaba Yaghoot is currently a co researcher in the Intelligent System and Robotic Lab at Iranian Institute of Advance Science and Technology (IRAN SSP) of the program for design high precision and fast dynamic controller for multi-DOF- actuators. 
International Journal of Hybrid Information Technology Vol.8, No.3 (2015) 